\title{
Utility of Correlation Measures in Analysis of Gene Expression
}

\author{
Anthony Almudevar,* Lev B. Klebanov ${ }^{\dagger}$ Xing Qiu,* Peter Salzman,* and Andrei Y. Yakovlev* \\ *Department of Biostatistics and Computational Biology, University of Rochester, Rochester, New York 14642; and ${ }^{\dagger}$ Department \\ of Probability and Statistics, Charles University, and Institute of Informatics and Control of the National Academy of Sciences, \\ Sokolovska 83, Praha-8, CZ-18675, Czech Republic
}

\begin{abstract}
Summary: The role of the correlation structure of gene expression data are two-fold: It is a source of complications and useful information at the same time. Ignoring the strong stochastic dependence between gene expression levels in statistical methodologies for microarray data analysis may deteriorate their performance. However, there is a host of valuable information in the correlation structure that deserves a closer look. A proper use of correlation measures can remedy deficiencies of currently practiced methods that are focused too heavily on
\end{abstract}

strong effects in terms of differential expression of genes. The present paper discusses the utility of correlation measures in microarray data analysis and gene regulatory network reconstruction, along with various pitfalls in both research areas that have been uncovered in methodological studies. These issues have broad applicability to all genomic studies examining the biology, diagnosis, and treatment of neurological disorders. Key Words: Gene expression, correlation, microarrays, genetic networks.

\section{INTRODUCTION}

The recent explosion in high-throughput technologies in genomics and proteomics opens up a new research area to the biostatistician. As more and more scientists and clinicians begin to use such technologies to generate new datasets, the need to establish well-designed studies with appropriate statistical methodologies is extremely pressing. This is especially true in the field of neurological disorders and neurotherapeutics, where other variables, including the complicated natural history of many of these diseases, other clinical variables, and current practical limitations in assessing biological function or dysfunction, also influence study design and interpretation.

There are two conflicting points of view regarding the role of correlation between gene expression levels that are clearly seen in the relevant methodological literature on microarray data analysis. In what follows, we will discuss the role of correlations solely in conjunction with high-density oligonucleotide gene expression arrays, although similar issues arise in analysis of gene expression data generated by other platforms. In numerous works

Address correspondence and reprint requests to: Andrei Yakovlev, Department of Biostatistics and Computational Biology, University of Rochester, 601 Elmwood Avenue, Box 630, Rochester, NY 14642. E-mail: Andrei_Yakovlev@urmc.rochester.edu. dealing with statistical methods for selecting differentially expressed genes, the strength of dependence between genes has been gravely underestimated. It is quite typical that the assumption of independence is explicitly or implicitly invoked in such works. Some authors proposed generalizations allowing for a weak stochastic dependence that preserves consistency of statistical estimators when pooling expression data across genes. This more general assumption is frequently used in conjunction with the empirical Bayes method and false-discovery rate (FDR) controlling procedures. For example, Storey ${ }^{1}$ advocates a form of weak dependence between genes, which he terms the "clumpy dependence." $\mathrm{He}$ hypothesizes that clumps of strongly interacting genes involve a small number of members and that each clump is independent of the others. As discussed in under The Correlation Structure of Microarray Gene Expression Data, this assumption lacks support from actual microarray data. An empirical study conducted by Qiu and coworkers ${ }^{2}$ also suggests that such clumps rather involve thousands of tightly dependent genes. The consequences of disregarding the strength of correlation in some methods of microarray data analysis are briefly discussed under Adverse Effects of Correlation between Genes.

The opposite tendency to overvalue the role of correlations is quite typical for works on pathway recognition and gene regulatory network reconstruction. For exam- 
ple, when building associative/relevance networks from gene expression data, the correlation or mutual information measures are frequently taken as characteristics of causal relationships or regulatory interactions between the genes. In other words, there is a tendency to endow correlation measures with a biological meaning they do not have. It is well known that additional stringent assumptions are necessary for the causal inference on an underlying network within the Bayesian framework. ${ }^{3}$ Yet another possibility is to analyze gene perturbation experiments that are specially designed to reconstruct relatively small networks of a simplistic structure. Time course gene expression data are also amendable to causal inference. By no means, however, may a graph built on correlation measures estimated from a set of "static" data be considered as an ultimate representation of betweengene interactions. We will expand on this issue under The Nature of Stochastic Dependence between Gene Expression Levels and the Conclusion.

Early in the development of methods for microarray analysis, it was conventional to declare a gene differentially expressed when its fold difference in two-group comparisons exceeds a certain cut-off level. This ad hoc selection rule is long gone and is now replaced with significance testing. The disadvantages of fold-difference measures calling for this conceptual change were discussed by Wolfinger and coworkers. ${ }^{4}$ Modern methods of microarray data analysis typically employ two-sample statistical tests for testing differential expression of genes combined with multiple testing procedures to guard against type 1 errors (see ${ }^{5-10}$ for reviews). Although a big step forward from the fold-difference criteria, such methods are still biased toward selecting those genes that display the most pronounced differential expression. It has become common practice to rank candidate genes using purely statistical criteria, such as $p$ values, and this ranking is thought to reflect their relative importance. This paradigm falls far short of being a perfectly valid approach because it is widely recognized that the magnitude of differential expression does not necessarily indicate biological significance. Even a very small change in expression of a particular gene may have dramatic physiological consequences if the protein encoded by this gene plays a catalytic role in a specific cell function. Many downstream genes may amplify the signal produced by this truly interesting gene, thereby increasing their chance to be selected by formal statistical methods. For a regulatory gene, however, the chance of being selected by such methods may diminish as one keeps hunting for downstream genes that tend to show much bigger changes in their expression. As a result, the initial list of candidates may be enriched with many effector genes that do not play any major regulatory role in the biological processes under study. That such an outcome is quite likely follows from a special type of stochastic dependence observed in microarray data and described under The Nature of Stochastic Dependence between Gene Expression Levels.

There are two natural ways to remedy the situation. One is to use bioinformatics tools that use prior biological knowledge, such as partially known pathways, for prioritization of candidate genes. This approach is now routinely used in biological studies and there are ongoing efforts to enrich it with specially designed algorithms. ${ }^{11}$ Another way is to extract additional information on relationships between different genes from microarray data by pertinent statistical methods. Both approaches enrich and supplement each other when used in combination.

Notwithstanding the importance of testing for differential expression of genes, we suggest that the whole concept of focusing solely on such genes be changed. Characteristics of higher dimension should be employed to initially prioritize candidate genes and provide the biologist with an additional source of information for decision making. At least some useful information can be extracted for this purpose by considering gene pairs in addition to individual genes and this is why a closer look at the correlation structure of gene expression data is the next natural step on the road to truly powerful methods of microarray data analysis. As larger sets of microarray gene expression data become more readily available, quantitative insights into dependencies between gene expression levels are gaining in importance (see The Nature of Stochastic Dependence between Gene Expression Levels and Useful Biological Information Hidden in Correlations). This paradigm is also instrumental in bridging the gap between the analysis of thousands of genes in microarray studies and the area of network inference, the latter being usually limited to a relatively small number of genes. The true potential of microarray technology and network analysis has yet to be unveiled (see Ma'ayan and coworkers ${ }^{12}$ ).

It is important to note that, although we will discuss statistical methodologies in high order data sets with respect to gene array (transcriptomic) studies in the cancer field, these methodologies should prove useful in a wide range of fields, including the study of neurological disorders and their treatments and therapies (see Ginsberg and coworkers ${ }^{13}$ and Miller and Federoff ${ }^{14}$ in this issue).

\section{THE CORRELATION STRUCTURE OF MICROARRAY GENE EXPRESSION DATA}

In this section, we provide quantitative information on the actual correlation structure of microarray data obtained by estimating correlation coefficients between gene expression levels from two sets of data on childhood leukemia. In doing so, we focus on two groups of patients with hyperdiploid (HYPERDIP) 
Hyperdip

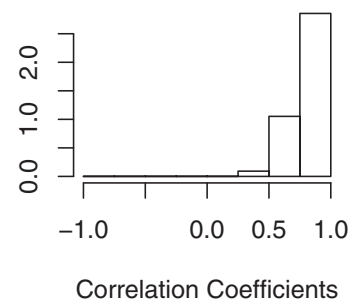

TEL-AML1

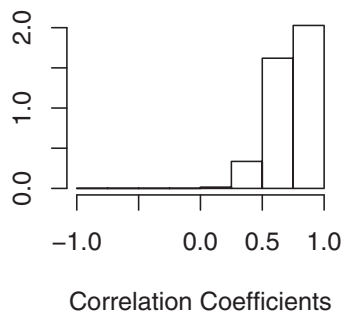

FIG. 1. Histograms of correlation coefficients for all pairs of gene in HYPERDIP (left) and TEL-AML1 (right).

acute lymphoblastic leukemia (88 patients) and a specific subclass of B lineage acute lymphoblastic leukemias (TEL-AML1) characterized by the $\mathrm{t}(12 ; 21)(\mathrm{p} 13$; q22) translocation (77 patients). Both groups of patients were identified through the publicly available St. Jude Children's Research Hospital (SJCRH) Database (http://www.stjuderesearch.org/data/ALL1/). Each high-density oligonucleotide array (Affymetrix, Santa Clara, CA) in the database reports expression measurements on $m=12,558$ probe sets.

Figure 1 displays the histograms of Pearson's sample correlation coefficients in all pairs of genes for each group of patients. It is clear that both histograms show the prevalence of high positive values of the correlation coefficients. The simplest explanation of this pattern is attributed to the random effect caused by a multiplicative array-specific technological noise. However, such an explanation is not consistent with a substantial proportion of independent pairs. Although it is possible to construct theoretical examples in which the presence of a multiplicative random noise may make a dependent gene pair stochastically independent, the abundance of independent pairs in the biological data speaks against this exotic possibility. Despite many "horror stories" told in methodological papers about the technological noise in microarray data, the actual level of this noise remains unknown due to the lack of well-designed metrological studies. Yet another underpinning for the abundance of high true correlations in microarray data are provided by a phenomenon described under The Nature of Stochastic Dependence between Gene Expression Levels.

Table 1 presents similar data on randomly selected individual genes. This table reports the number of pairs formed by a given gene with all other genes whose correlation coefficients are within a certain range. The table shows the presence of a "long-range" and extremely high correlation involving thousands of genes. However, there are also genes that are practically independent of all other genes as discussed in the next section.
TABLE 1. The Frequency of Correlation Coefficients in Pairs Formed by 10 Randomly Selected Genes with All Other Genes

\begin{tabular}{lrrrr}
\hline & & $(0.25$, & $(0.5$, & \\
& $<0.25$ & $0.5)$ & $0.75)$ & $\geq 0.75$ \\
Gene 1 & 4 & 513 & 6255 & 5786 \\
Gene 2 & 9 & 139 & 6402 & 6008 \\
Gene 3 & 81 & 1867 & 5448 & 5162 \\
Gene 4 & 47 & 4698 & 7791 & 22 \\
Gene 5 & 7 & 93 & 4169 & 8289 \\
Gene 6 & 213 & 2934 & 8200 & 1211 \\
Gene 7 & 13 & 114 & 2284 & 10147 \\
Gene 8 & 133 & 3986 & 7076 & 1363 \\
Gene 9 & 5 & 77 & 4950 & 7526 \\
Gene 10 & 12 & 2321 & 8222 & 2003 \\
\hline
\end{tabular}

The estimates of correlation coefficients are obtained from the HYPERDIP group of the SJCRH dataset.

\section{THE NATURE OF STOCHASTIC DEPENDENCE BETWEEN GENE EXPRESSION LEVELS}

Using three large sets of microarray data on childhood leukemia, Klebanov and coworkers ${ }^{15}$ studied the expression profiles of all pairs of genes formed from a total of 12,558 genes (probe sets). The authors discovered a very special type of stochastic dependence between genes, the existence of which was later corroborated by similar analyses of some smaller data sets. ${ }^{15}$ To characterize this type of stochastic dependence, let $X$ and $Y$ be random variables representing the expression levels of genes $g_{x}$ and $g_{y}$. A pair of genes $\left(g_{x,}, g_{y}\right)$ is said to be of Type A if $X$ and $Y$ satisfy the condition:

$$
Y=X Z
$$

where $Z$ is a positive random variable that is stochastically independent of $X$. All random variables in Eq. (1) are defined on the same probability space. The mean value of $Z$ can be greater or smaller than 1 . It follows from the above definition that the random variables $X$ and $Y / X$ are independent in a Type A gene pair. At the same time, the random variables $X$ and $Y$ are stochastically dependent so that the regular correlation between them can be quite strong. The roles of $g_{x}$ and $g_{y}$ in such a pair are not symmetrical because, given Eq. (1) is true, the random variables $Y$ and $1 / Z$ in the relationship $X=$ $Y / Z$ are no longer independent. This makes the relationship in Eq. (1) unidirectional and gives us the reason to call $g_{x}$ the "driver" and $g_{y}$ the "modulator."

The relation (1) is directly discerned in gene expression data via testing independence of $X$ and $Y / X$ by a statistical test specially designed to allow for an arrayspecific random noise. ${ }^{15} \mathrm{~A}$ total of more than $35 \%$ of all unordered pairs formed from 12,558 genes were conservatively estimated to belong to Type A and further stud- 


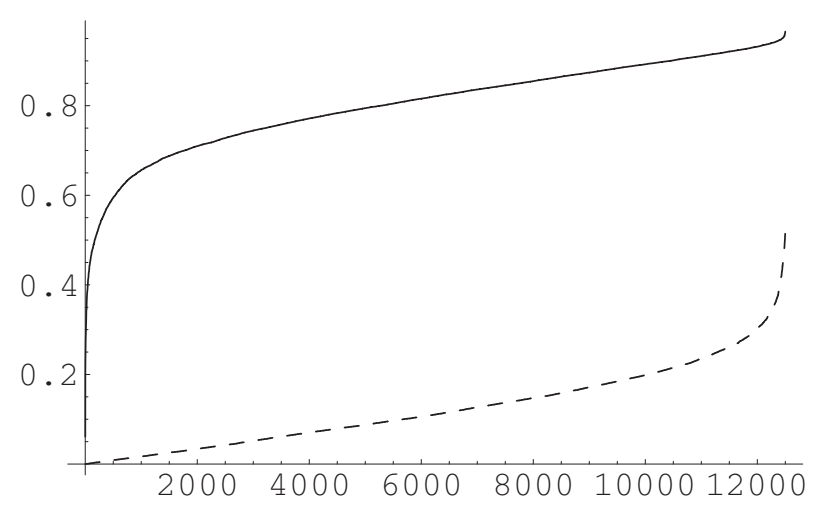

FIG. 2. Type A dependence prevails in gene pairs formed by this gene (WNT10B) because the correlation between $X$ and $Y / X$ (lower line) is lower than 0.2 for more than 10,000 pairs. The correlation between $X$ and $Y$ (upper line) is high for the majority of pairs formed by WNT10B. $x$ axis: ordered pair number; $y$ axis: correlation coefficient.

ies have shown that the presence of Type A pairs represents a remarkably stable mass phenomenon that cannot be explained by a multiplicative random noise in the data. In this connection, it is important to note that the random variable $Z$ has dissimilar distributions in different (and numerous) gene pairs. Type A pairs form long sequences (or chains) involving thousands of genes. Such chains of genes may change their structure (composition) under different pathological conditions.

It is important to emphasize once again that the dependence of Type A is a strong stochastic dependence. For illustration purposes, we use a subset of microarray data on 88 patients with hyperdiploid acute lymphoblastic leukemia identified through the SJCRH Database. Shown in Figure 2 are the Pearson correlation coefficients in all pairs formed by gene WNT10B in increasing order. The (upper line) in Figure 2 indicates a very strong correlation of the selected gene with the overwhelming majority of other genes. To see whether this strong dependence is in fact a manifestation of Type A dependence, we compute correlations between $X$ and $Y / X$ and between $Y$ and $X / Y$, respectively, and take the minimal of the two. The resultant correlation coefficients for all pairs formed by gene WNT10B are also plotted in increasing order (lower line). It is clear that the latter correlation coefficients tend to be quite small and therefore the selected gene forms Type A relationships with the majority of genes.

While not ruling out the possibility of causal relationships in Type A gene pairs, Klebanov and coworkers ${ }^{15}$ give a cautious interpretation of observed chains of Type A pairs as "supply chains" that provide certain proportions of gene products to fulfill a specific cell function. This interpretation suggests that genes involved in Type A pairs appear to act as effector rather than regulatory genes. When comparing two groups of patients with leukemia, the authors found a total of 342 differentially expressed genes. They note that a subset of genes with smallest $p$ values is dramatically enriched (47 of 50) with genes forming Type A relationships with other genes that are declared statistically significant in terms of differential expression. This subset, comprised mostly of modulators, is enriched with signal transduction factors. The frequency of transcription factors is significantly reduced in this subset compared with a subset of 49 genes selected by removing all modulators from the set of differentially expressed genes. This finding is consistent with the above interpretation of Type A dependencies as relationships between effector genes.

Those gene pairs that do not satisfy condition (1) are classified as Type B pairs. Unlike Type A pairs, the pairs of Type B do not imply the asymmetric dependence mentioned above. Despite the fact that Type B pairs usually display low correlation between their members they are likely candidates for a regulatory function because the absence of correlation is readily consistent with the well-known modes of regulation such as induction (promotion) and repression. Another caveat is that building relevance networks exclusively on correlations ${ }^{16}$ may lead to a graph displaying predominantly Type A relationships between the genes selected by thresholding correlation coefficients.

\section{ADVERSE EFFECTS OF CORRELATION BETWEEN GENES}

A characteristic feature of the correlation structure of microarray gene expression data is a "long-range" correlation that manifests itself in an abundance of large sets of heavily dependent genes (see under The Correlation Structure of Microarray Gene Expression Data and The Nature of Stochastic Dependence between Gene Expression Levels). Such sets may involve thousands of genes and this feature persists even in the data normalized by different methods. ${ }^{2}$ It is clear that this type of correlation contraindicates pooling expression measures, or associated test statistics, or $p$ values across genes, an expedient widely used by many statistical methodologies such as the empirical Bayes method or other procedures controlling the FDR. The empirical Bayes method attempts to overcome the notorious cost and labor limitations of microarray experiments by treating test statistics associated with each gene as a sample drawn from some distribution. The method requires estimation of the distribution density for a chosen test statistic (usually the $t$ statistic) from observed values of this statistic for all genes, the latter values being treated as a random sample. However, it is important that the test statistics associated with different genes are not independent random variables as discussed under The Correlation Structure of Microarray Gene Expression Data. Yet another, though relatively minor, concern is that even pivotal test statis- 
tics, such as the $t$ statistic, are not identically distributed in the presence of differentially expressed genes. If one still treats the observed test statistics for different genes as a sample of independent and identically distributed (i.id) random variables, the price to be paid is an extremely high variance of the length (and composition) of the list of genes declared differentially expressed. ${ }^{17}$ Other performance indicators such as the estimated FDR suffer from the same kind of instability as well. ${ }^{17,18}$ The high correlation between test statistics can induce biases in some performance indicators as well. Furthermore, even consistency of the traditional statistical estimators (for which this property is well established in the case of i.id or weakly dependent observations) becomes questionable. Consistency is the most fundamental property of a statistical estimator that guarantees its convergence to the true parameter in large samples.

Qiu and coworkers ${ }^{2}$ addressed the consistency question in terms of the $t$ statistics associated with each gene by analyzing the SJCRH Database on childhood leukemia. In this analysis, a total of 330 arrays ( 1 array per subject), each reporting measurements on the same set of $m=12,558$ genes, were randomly split into 30 parts, each containing 11 arrays. Fifteen pairs of the array subsamples then were arranged and the corresponding 15 $t$ statistics were computed for each gene, thereby mimicking 15 two-sample comparisons under the null hypothesis of no differential expression. As a result, each gene was associated with 15 values of the $t$ statistic and the input for analysis was a $12,558 \times 15$ matrix of observed $t$ statistics. The authors designed the following algorithm:

1. Select randomly a certain number $v$ genes and compute the arithmetic (sample) mean of the $t$ statistics across these genes for each pair of subsamples.

2. Compute the standard deviation of the sample mean across the pairs of subsamples.

3. Select randomly $v$ from the remaining genes and compute the arithmetic mean for the $2 v$ genes for each pair of subsamples.

4. Compute the standard deviation from the sample means resulted from the previous step.

5. Continue until the set of all genes is exhausted.

6. Plot the standard deviation as a function of the number of genes involved in each step of the algorithm.

7. Repeat the procedure $l$ times to generate $l$ trajectories of the standard deviation.

The authors conducted this experiment with $v=100$ and multiple random starts. It is well known that the sample mean is an unbiased and consistent estimator for the true mean value in the case of i.id observations. In this case, the standard deviation decreases very rapidly and tends to zero with increasing the number of genes. However, the same was not true for the actual biological data. For the unnormalized data, the standard deviation did not show a tendency to decrease at all. When the data were normalized using the quantile normalization procedure, the standard deviation dropped initially and then stabilized at an approximately constant level, no matter how many genes were involved in estimation of the mean value. The pattern seen in the biological data suggests that the estimator is likely to converge to a random variable rather than to the true parameter to be estimated. The authors attributed this effect to the strong correlations between the $t$ statistics that manifest themselves in gene expression data.

The presence of the long-range amplification-like dependence described under The Correlation Structure of Microarray Gene Expression Data sheds additional light on the nature of the adverse effects of correlations described above. Pooling such observations may cause huge losses rather than gains in statistical information contained in the sample even in the case of identically distributed observations. The latter statement can be illustrated with an extreme example where the expressions of all genes take on the same values with probability one, thereby providing only the information contained in the expression profile of a single gene.

\section{USEFUL BIOLOGICAL INFORMATION HIDDEN IN CORRELATIONS}

It is noteworthy that recent years have seen a growing interest in correlations between gene expression levels in statistical methodologies for microarray analysis. ${ }^{2,17,19-24}$ The correlation coefficient has been used extensively as a measure of similarity in gene clustering since a seminal paper by Eisen and coworkers ${ }^{25}$ The material of The Correlation Structure of Microarray Gene Expression Data and The Nature of Stochastic Dependence between Gene Expression Levels elucidates the type of correlation structure one is dealing with when analyzing microarray data. It also shows that the correlation structure of microarray data is an important indicator of changes in transcription profiles between two phenotypes.

A recent paper by Dettling and coworkers ${ }^{23}$ is the first attempt to explore this possibility. The authors proposed statistical tools for testing changes in correlation coefficients in all gene pairs when comparing two different phenotypes. In particular, they proposed the following score function:

$$
S_{i}=\left|r_{1 i}+r_{2 i}-\alpha r_{i}\right| \text {. }
$$

where $r_{1 i}$ is the Pearson sample correlation coefficient of the $i$ th pair of genes in Phenotype 1, $r_{2 i}$ is the Pearson 

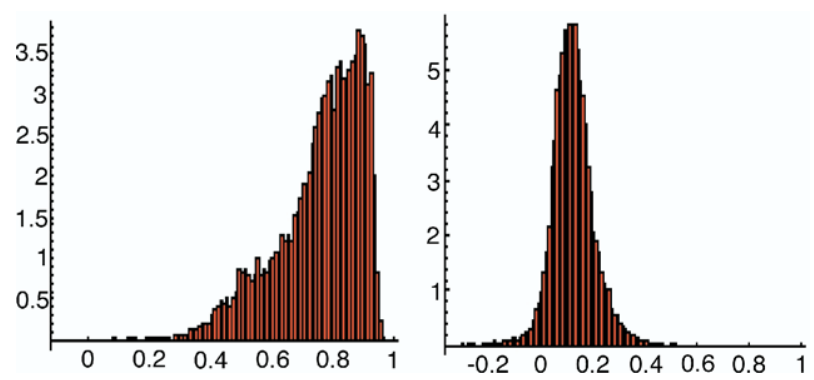

FIG. 3. Histograms of correlation coefficients computed for all gene pairs formed by a particular gene. Observed differences between TALL (left) and HYPERDIP (right) are quite pronounced in this case. $x$ axis: correlation coefficient; $y$ axis: frequency.

correlation coefficient for the same pair identified in Phenotype 2, $r_{i}$ is the unconditional sample correlation coefficient derived from the pooled sample, and $\alpha$ is a tuning parameter. This scoring function appears to be sensitive to special types of joint differential expression that the authors call "substitution" and "gap" cases. Based on the score, Eq. (2), the authors propose a permutation test designed to select pairs of genes with statistically significant changes in the correlation between their expression levels. However, they do not address the multiple testing aspect of this problem. Under the socalled "on-off" scenario, the authors recommend using a slightly different score:

$$
\tilde{S}_{i}=\left|r_{1 i}-r_{2 i}\right| \text {. }
$$

They also consider the utility of logistic regression for the same purpose. The usefulness of their approach is illustrated with an application to two sets of biological data and computer simulations. In particular, they provide examples of gene pairs for which a marked difference in correlation coefficients is not accompanied by a significant difference in the marginal distributions of expression levels for both genes. In our opinion, this paper is a forerunner of forthcoming conceptual changes in modern methodological approaches to microarray data analysis.

The question arises as to how many genes are expected to change their correlations with other genes in real world applications. Some genes display dramatic changes in the vector of correlation coefficients (Figure 3 ). At the same time, such changes appear to be small for other genes (Figure 4). The histograms in Figures 3 and 4 were constructed from the SJCRH data on patients with T-cell acute lymphoblastic leukemia (TALL) and hyperdiploid acute lymphoblastic leukemia (Hyperdip). These figures suggest that testing equality of correlation vectors across different phenotypes may provide an additional source of useful information for prioritizing candidate genes. To put this idea to practical use would require developing new, sophisticated methods for multivariate comparison of huge correlation vectors. This represents a
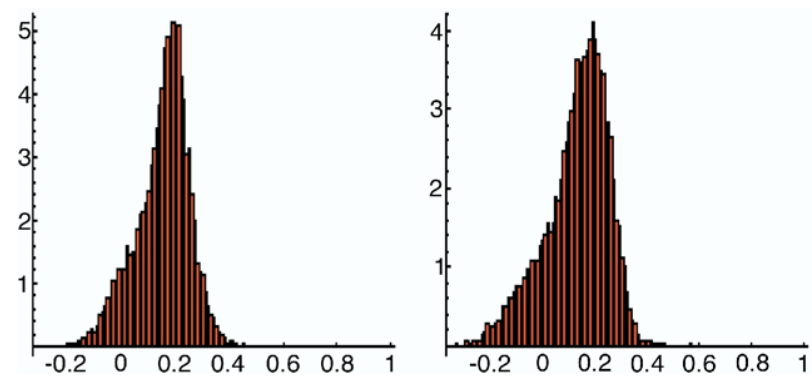

FIG. 4. Histograms of correlation coefficients computed for all gene pairs formed by another gene. Observed differences between TALL (left) and HYPERDIP (right) are small in this case (compare with FIG. 3). $x$ axis: correlation coefficient; $y$ axis: frequency.

very promising research area and such studies are currently underway in our laboratory.

\section{SAMPLE SIZE ISSUES AND USEFULNESS OF MICROARRAY TECHNOLOGY}

As it stands, the microarray analysis by itself produces too many artifacts to be fully appreciated by biologists. This is a price to be paid for inadequate sample sizes traditionally used in microarray studies. For a mysterious reason, it took a long time for researchers to realize that microarray experiments must be replicated. ${ }^{26}$ Now it is time to realize that the sample size in such studies must be vastly larger. It is still common to report microarray data on a small number (3-10 per group) of arrays (subjects). To better appreciate the inadequacy of this sample size, one can think of a clinical or experimental trial enrolling a similar number of subjects to test the effect of a specific drug on just a single endpoint. It is difficult to envision this small sample size to result from a pertinent power calculation required by the well-established policies for such studies. The situation is largely similar when focusing on a single gene in microarray analysis and it is even exacerbated by the need to conduct tens of thousands of tests simultaneously.

Contrary to popular belief, we think that the requirements to be imposed on the sample size in microarray studies have little to do with the usual power calculations, because the analysis of microarrays is exploratory (not confirmatory) by its very nature and the expected effect sizes for different genes are usually unknown. To the best of our knowledge, no theoretically sound methodology has been proposed to do power calculations in the presence of multiple tests. Therefore, recommendations on this issue should be general enough to cover a wide range of possible situations. It is only the experience accumulated from numerous analyses of various data sets that can suggest a reasonable range of sample sizes. Pavlidis and coworkers ${ }^{27}$ proposed to approach the sample size problem from the selection stability perspec- 
tive and we find their idea very interesting and deserving of further development.

Microarray experiments are notoriously expensive. This circumstance imposes tight constraints on the available sample size, driving statisticians to look for artificial ways to overcome sample size limitations by pooling information across genes. However, the strong correlation between gene expression levels presents a serious (and probably insurmountable) obstacle to the application of such methods (Adverse Effects of Correlation between Genes). We are convinced that there is no alternative to increasing the sample size that is determined by the number of subjects under study rather than by the number of genes. The main challenge of microarray data analysis is usually referred to as the "large dimension, small sample size" problem. However, the most fundamental question is: How small is small? Our simulation studies suggest that some traditional testing procedures controlling the family-wise error rate seem to do a good job (in terms of the stability of gene selection and overall power) with such moderate sample sizes as $40-50$ (arrays) per group. ${ }^{2}$ By generating samples of this size (or larger!), it will become possible to more widely use distribution-free statistical tests that offer a great advantage in situations in which the exact unadjusted $p$ values are required as input for multiple testing procedures. ${ }^{28-31}$ With a larger sample size one also gains the possibility to provide quantitative insight into the correlation structure of gene expression that conveys important biological information far beyond the marginal measures of differential expression.

By saving biological replicates needed for selecting differentially expressed genes, one takes huge losses of time and money when trying to validate irrelevant findings. The price for missing biologically important candidates is difficult to estimate. However, the most tangible price we pay for insufficient sample sizes is that the real potential of microarray technology remains untapped. The microarray technology yields abundant multidimensional information on the functioning of the whole genome machinery at the level of transcription so that much can be learned about interrelationships between genes and mechanisms by which the cell assigns tasks to different genes to maintain a specific function. In particular, a host of useful information can be extracted from the correlation structure of gene expression signals. It is unfortunate that such an advanced technology continues to be used as a simplistic screening tool with a focus on big differences between mean values of expression measurements. The future of this and similar technologies, and thus of the biology as a whole, hinges on our ability to generate much larger samples. It is now clear that the high cost of microarray chips stymies the progress of biological sciences. This problem cannot be fixed mathematically and it is extremely important that both the industry and the scientific community take the above-voiced concern very seriously and work together to fully use the abundance of information yielded by microarray studies.

We believe that the issues we have discussed are important to the design, data collection, analysis, and interpretation of all genomic studies examining the biology, diagnosis, and treatment of neurological disorders.

\section{THE USE OF COEXPRESSION MEASURES IN DETERMINING GENE FUNCTION}

The most natural application of coexpression measures is in the determination of gene function. Suppose a set of genes is involved in a common process. If we obtain a sample of microarray slides we would expect to see the expression levels of this set exhibit mutually higher or lower expression levels in a coordinated manner across the sample. This forms the basis for the reconstruction of regulatory relationships from microarray data and is quite distinct from the more common practice of associating differential gene expression with an experimental treatment.

Underlying most forms of functional inference is some combinatorial object. The simplest is a partition, which assigns each gene to a distinct class, on the basis of some similar pattern of gene expression (see Eisen and coworkers $^{25}$ ). Microarrays are sampled under some fixed number $K$ of experimental conditions designed to induce differing gene expression profiles. Each group is pooled, resulting in a $K$ dimensional feature vector for each of $N$ genes. A distance (or similarity) measure is applied, resulting in an $N \times N$ distance matrix, which can be input to any statistical clustering method (see Baldi and Hatfield $^{32}$ for a good summary). Note that such a distance need not be a useful measure of coexpression, although measures of coexpression can be transformed into distances. Although such partitions may be useful in a variety of applications, such as prognostic signatures, as representations of functional relationships they are quite coarse, and a more refined estimate of functional relationship should be possible.

\section{Graphical representation of coexpression}

Most representations of gene interactions take the form of a graph in which nodes represent genes, and two nodes are connected by an edge if the two genes interact. An edge between nodes $a$ and $b$ is directed (undirected) if the relationship is ordered (unordered). A graph is directed (undirected) if all edges are directed (undirected). A graph may have both types of edges. A directed edge between $a$ and $b$ is directed either from $a$ to $b(a \rightarrow b)$ or $b$ to $a(b \rightarrow a)$. Given edge $a \rightarrow b$ we say that $a$ is a parent of $b$, and $b$ is an offspring of $a$. Then $a$ is an ancestor of $d$ if there is a directed path from $a$ to $d$, in which case a 
transitive causal relationship exists between $a$ and $d$ if they are not connected by a single edge. In the context of gene networks, the edge $a \rightarrow b$ implies that gene $a$ regulates $b$ in the sense that the expression (or suppression) of $b$ is a direct consequence of the expression of $a$. If gene $a$ were, for example, deleted, this would affect the expression levels of $b$, but a deletion of $b$ would not affect the expression of $a$. There is, therefore, a very definite biological interpretation that can be assigned to the direction of an edge. The undirected edge $a-b$ is not as informative. It implies the existence of an interaction between $a$ and $b$ but says nothing about the direction of regulation.

To clarify exactly what information is contained in a graph, consider three genes $a, b$, and $c$. Suppose $a$ regulates $b$ and $b$ regulates $c$. A directed graph representing this situation would presumably include edges $a \rightarrow b$ and $b \rightarrow c$ To decide whether to include edge $a \rightarrow c$, we ask whether $a$ would regulate $c$ in the absence of $b$. If not, then $a \rightarrow c$ should not be included. The same issue would be relevant if we wished to use an undirected graph.

Now suppose we accept that a gene expression level is a random variable and there exists a joint distribution $f$ across all genes. The question of whether an edge exists between two genes can be expressed with mathematical formality by the notion of conditional independence. Suppose the gene expression levels of $a, b$, and $c$ obey the following system:

$$
\begin{aligned}
& X_{b}=f_{1}\left(X_{a}\right)+\varepsilon_{1} \\
& X_{c}=f_{2}\left(X_{b}\right)+\varepsilon_{2}
\end{aligned}
$$

where $\varepsilon_{1}, \varepsilon_{2}$ are independent random variables. Here, $X_{a}$ and $X_{c}$ are independent conditional on $X_{b}$. To see this, suppose we are given the realized value of $X_{b}$. To determine $X_{a}$ we need only $\varepsilon_{1}$ and to determine $X_{c}$ we need only $\varepsilon_{2}$, and these two quantities are independent. We could then represent this system as $a \rightarrow b \rightarrow c$. The conditional independence of $X_{a}$ and $X_{c}$ is expressed by the fact that $b$ separates $a$ and $c$ in the sense that it blocks the only path from $a$ to $c$.

This leads to a notion of a gene interaction graph as a representation of the conditional independencies of $f$. An understanding of some preliminary theory is needed to determine how to turn statistical measures of coexpression into statements about gene interaction. The following definitions will form a useful starting point:

Definition 1 (Global Markov Property for Undirected Graphs). Suppose $G$ is an undirected graph with nodes $1, \ldots, N$. Let $x, y$ be distinct nodes, and let $Z$ be a set of nodes not containing $x$ or $y$. Possibly, $Z$ is empty. We say $x$ and $y$ are separated by $Z$ if all paths from $x$ to $y$ contain at least one node from $Z$. If $X, Y, Z$ are disjoint sets of nodes, with $Z$ possibly empty, we say $X$ and $Y$ are separated by $Z$ if all pairs of nodes $(x, y) \in X \times Y$ are separated by $Z$.

Now suppose a random vector $\widetilde{U}=\left(U_{1}, \ldots, U_{N}\right)$ possesses distribution $f$, and $U_{j}$ labels node $j$. Let $\widetilde{U}(X)$ be those components of $\widetilde{U}$ that label nodes in $X$. The pair $(f, G)$ satisfies the global Markov property for undirected graphs if $\widetilde{U}(X)$ and $\widetilde{U}(Y)$ are independent conditional on $\widetilde{U}(Z)$ whenever $X$ and $Y$ are separated by $Z$. When $Z$ is empty we take this to imply unconditional independence of $\widetilde{U}(X)$ and $\widetilde{U}(Y) . / / /$

A similar definition exists for directed graphs. Although formally more complicated, it also relies on the notion of conditional independence being induced by genes that block paths.

Definition 2 (Global Markov Property for Directed Graphs). Suppose $G$ is a directed graph with nodes $1, \ldots, N$. Let $x, y$ be distinct nodes, and let $Z$ be a set of nodes not containing $x$ or $y$. Possibly, $Z$ is empty. All nodes on an undirected path from $x$ to $y$ can be classified as endpoints ( $x$ and $y$ ), colliders (nodes pointed into by both adjacent nodes), or noncolliders. We say $x$ and $y$ are $d$-separated by $Z$ if at least one non-collider is in $Z$ or there exists at least one collider that is not an ancestor of any node in $Z$. If $X, Y, Z$ are disjoints sets of nodes, with $Z$ possibly empty, we say $X$ and $Y$ are $d$-separated by $Z$ if all pairs of nodes $(x, y) \in X \times Y$ are $d$-separated by $Z$.

Now suppose a random vector $\widetilde{U}=\left(U_{1}, \ldots, U_{N}\right)$ possesses distribution $f$, and $U_{j}$ labels node $j$. Let $\widetilde{U}(X)$ be the components of $\widetilde{U}$ which label nodes in $X$. The pair $(f, G)$ satisfies the global Markov property for directed graphs if $\widetilde{U}(X)$ and $\widetilde{U}(Y)$ are independent conditional on $\widetilde{U}(Z)$ whenever $X$ and $Y$ are $d$-separated by $Z$. When $Z$ is empty we take this to imply unconditional independence of $\widetilde{U}$ $(X)$ and $\widetilde{U}(Y)$. ///

This definition provides an intuitive connection between functional interactions between genes and their graphical representation. Suppose we accept the existence of a probability distribution $f$ governing a vector of gene expression levels $\widetilde{U}=\left(U_{1}, \ldots, U_{N}\right)$. The causal nature of gene interactions induces conditional independence between genes that interact only through intermediate regulation. These conditional independencies constrain $f$ and may be usefully represented by graphical models.

\section{Causality versus coexpression}

We can take coexpression between two genes to be a consequence of their location on the same pathway. An undirected graph tells us whether two genes interact directly, which will be considerably more informative than a cluster partition, but will not establish the direction of 
causality, which is necessary in determining those genes responsible for initiating a process. This leaves the question: To what degree can we determine causality using measures of coexpression? The objective is to determine a directed graph $G$, which satisfies the global Markov property with gene distribution $f$. Unfortunately, such a graph will not generally be unique. Suppose we confine attention to directed acyclic graphs (DAGs), which are directed graphs that contain no cycles (directed paths which start and end at the same node). Two DAGs are called equivalent if they imply the same set of conditional independencies, and graphs that are equivalent form equivalence classes.

Suppose we can estimate features of $f$ within a reasonable statistical error, in particular, statistical dependence between genes as well as conditional independence. We would then be able to deduce a graph satisfying the global Markov property with $f$ up to an equivalence class. The following theorem, from Verma and Pearl, ${ }^{33}$ states exactly to what extent causality can be deduced from this type of inference.

Theorem 1. Two DAGs are equivalent if and only if they have identical topologies (undirected graphs) and $(a, b, c)$-structures (node triples of the form $a \rightarrow b c$ ). I//

Because we can identify topologies, we can distinguish between direct and transitive gene interaction. However, the determination of causality is more problematic. For example, consider genes $a, b, c$. Suppose we estimate the topology to be $a-b-c$. There are four directed graphs compatible with this topology:

$$
\begin{aligned}
& G_{1}=a \rightarrow b \rightarrow c \\
& G_{2}=a \leftarrow b \leftarrow c \\
& G_{3}=a \leftarrow b \rightarrow c \\
& G_{4}=a \rightarrow b \leftarrow c
\end{aligned}
$$

Of these, $G_{1}, G_{2}, G_{3}$, have no $v$ structure, and so we have two equivalence classes $\left\{G_{1}, G_{2}, G_{3}\right\}$ and $\left\{G_{4}\right\}$. This means we may only decide on a statistical basis whether the graph is $G_{4}$. Under $G_{4}$, by the definition of the global Markov property, genes $a$ and $b$ are independent $(b$ is a collider that is not an ancestor of any node in $Z$ if it is empty). This implies that genes $a$ and $c$ independently regulate $b$, a very different control law than that implied by $G_{1}, G_{2}$, or $G_{3}$.

In summary, a control law may or may not be statistically resolved by measures of coexpression and independence, the determinability being a property of the control structure itself. Furthermore, the level of determinability can be established by a consideration of equivalence classes generated by the global Markov property.

\section{Correlation and conditional independence}

The Pearson's moment correlation is a measure specifically of linear association, so that a value of 0 does not in general imply independence. It does, however, for multivariate normal random variables; hence, when this assumption is reasonable, small correlation can usually be taken to imply small levels of dependence. Furthermore, the determination of conditional independence is especially convenient in this case. Given random variables $X, Y$, and random vector $Z$, the partial correlation coefficient $r(X, Y \mid Z)$ is defined as the correlation of the pair $(X, Y)$ after controlling (linearly) for $Z$. This can be calculated by regressing $X$ and $Y$ separately on $Z$, then setting $r(X, Y \mid Z)$ equal to the correlation coefficient of the resulting residuals. If $(X, Y, Z)$ is a multivariate normal vector, zero partial correlation implies conditional independence. It is useful to classify partial correlation coefficients by order, taken to be the dimension of $Z$. The zero order partial correlation coefficient is simply the unconditional correlation. Letting $Z=\left(Z_{1}, Z_{2}\right)$, the first and second order sample partial correlation coefficients are given by

$$
r\left(X, \quad Y \mid Z_{1}=\frac{r(X, Y)-r(X, Z) r(Y, Z)}{\sqrt{\left(1-r(X, Z)^{2}\right)\left(1-r(Y, Z)^{2}\right)}}\right.
$$

$$
\begin{aligned}
r\left(X, Y \mid Z_{1}, Z_{2}\right) & \\
& =\frac{r\left(X, Y \mid Z_{2}\right)-r\left(X, Z_{1} \mid Z_{2}\right) r\left(Y, Z_{1} \mid Z_{2}\right)}{\sqrt{\left(1-r\left(X, Z_{1} \mid Z_{2}\right)^{2}\right)\left(1-r\left(Y, Z_{1} \mid Z_{2}\right)^{2}\right)}} .
\end{aligned}
$$

This provides a general method for reconstructing a graph that satisfies the global Markov property with respect to the gene expression distribution. We include an edge between genes $x$ and $y$ if the corresponding gene expression levels $X, Y$ satisfy $r(X, Y) \neq 0$ and if there is no other set of genes with expression vector $Z$ for which $r(X, Y \mid Z)=0$. In practice these conditions take the form of a suitable hypothesis test or threshold rule, usually empirically based, therefore one must account for the problems inherent in multiple testing. One potential drawback is that the number of sets of genes on which to test for conditional independence is equal to $2^{N-2}-1$. Fortunately, a number of algorithms based on this method have been implemented, reporting that, in practice, only low order partial correlation coefficients need be examined (see de la Fuente and coworkers ${ }^{34}$; Schafer and Strimmer ${ }^{35}$; Wille and Bühlmann ${ }^{36}$ ).

Note, however, that many forms of dependence have been reported in this type of application (Butte and Kohane $^{37}$ ), so that correlation-based methods may fail to detect some types of gene interaction. An important issue arises from the fact that a single microarray slide is usually made from the aggregate of a large number of cells, therefore, a single gene expression is really the sum 
of many independent processes. The correlation coefficient will be the same for the sum as for the individual processes, but the same is not in general true for conditional independence. See Chu and coworkers ${ }^{38}$ and Bahcall $^{39}$ for a discussion of this issue.

A simpler alternative to the use of partial correlation coefficients is to construct an edge only between gene pairs with an absolute correlation larger than some fixed threshold. We expect gene pairs with a transitive relationship to have a smaller correlation than those that interact directly, so that the threshold should act as a type of filter. Such an approach was proposed to create relevance networks in Butte and Kohane ${ }^{37}$ (but using an entropy-based measure of pairwise coexpression). These are used to generate hypothetical functional relationships in graphical form.

A different application of this type of procedure was proposed by Zhou and coworkers. ${ }^{40}$ An edge is accepted if the correlation measure is above a threshold. The correlation is transformed into a distance $1-|r|^{k}$, which then labels the edge. A hypothetical pathway fragment is generated by determining the shortest path between a given pair of genes, with the objective of associating open reading frames with genes of known function.

\section{GRAPHICAL MODELING}

Many proposed techniques for fitting graphical models are based on the idea of treating the graph itself as a parameter, rather than inducing a graph by estimating coexpression and conditional independence conditions. Typically, a score $\phi(G, X)$ is devised, which is a function of a graph $G$ and the collective data $X$, and measures the plausibility of graph $G$ in light of the data. This type of analysis, in its simplest form, accepts as an estimate of the true graph that $\phi(G, X)$. which maximizes (or minimizes) $\phi(G, X)$. The score can be a Bayesian posterior density

$$
\phi(G, X)=P(G \mid X)=\frac{P(X \mid G) P(G)}{P(X)}
$$

in which $P(X)$ is a normalizing constant, which usually need not be known, $P(X \mid G)$ can be calculated directly as a consequence of the model used, and $P(G)$ is a prior distribution on the set of all graphs considered. The selection of this prior is important when fitting graphical models. The conditional probability $P(X \mid G)$ will typically increase with the complexity of $G$ (the term overfitting applies here). If a uniform prior is used, the score will reward complexity, and the estimated graph will contain many spurious edges, so priors that penalize complexity are commonly used. It is also possible to use the prior to weight graphs according to scientific expectation. For example, it is more likely that a small number of genes regulates a large number of targets than vice versa. We may therefore adopt a prior that assigns greater weights to graphs that conform to these expectations. Similar considerations usually apply to scores that are not explicitly constructed as Bayesian posterior densities (see Jordan ${ }^{41}$ for a recent survey of this topic).

One further advantage of this approach is that model uncertainty can be assessed in a direct manner. If $\phi$ is interpreted as a posterior density (whether derived as such), computational Bayesian methods can be used to sample graphs from $\phi$. A confidence level for any feature of a graph may then be estimated as the proportion of sampled graphs that possess that feature, a process usually referred to as Bayesian averaging. These methods are often challenging to implement but have been consistently shown to be effective and remain a very active area of research (Madigan and York, ${ }^{42}$ Hoeting and coworkers ${ }^{43}$ Friedman and Koller, ${ }^{44}$ Dash and Cooper, ${ }^{45}$ Almudevar and Salzman ${ }^{46}$ ). In particular, one avoids the problem of multiple testing that arises in any edge selection method.

\section{Bayesian networks}

One of the most commonly used types of graphical model is the Bayesian network. Originally designed to model expert systems in artificial intelligence applications $\left(\right.$ Pearl ${ }^{47}$ ), it have been more recently applied to the problem of modeling gene regulatory networks (Friedman and coworkers, ${ }^{3}$ Freidman, ${ }^{48}$; Sebastiani and coworkers, ${ }^{49}$ Hartemink ${ }^{50}$ ). The Bayesian network model consists of a DAG of nodes $G$ labeled by gene expressions, with a joint distribution $f$ constrained by the conditional independencies implied by $G$ through the global Markov property for directed graphs. This permits a convenient form of $f$. Let $P a_{G}(X)$ be the set of parents of gene $X$ in network $G$. The model may then be described entirely by the influence of the parents on their offspring:

$$
f=\prod_{i} P_{\beta(G)}\left(X_{i} \mid X_{P a_{G}(X i)}\right) .
$$

Given knowledge of the network, in particular of all the parent sets, we can estimate the parameters $\beta(G)=\left\{\beta_{P a(X)}: i\right\}$ for all genes $i$. We use the likelihood principle and find the maximum likelihood estimator (MLE) conditional on the network.

The harder question is how to find the network. First, we must note that the problem at hand is a model selection problem. In particular, for each gene $i$ we look for the subset of genes that best explains $X_{i}$. Theoretically, we could find the likelihood $L(G)$ for each network and choose the best one. However, it is known that the likelihood of the network increases as the number of parents of each gene increases, leading to the problem of overfitting. Hence a score that controls graph complexity must be used. Among the most popular scores are the 
Bayesian Dirichlet equivalent (BDe), Akaike Information Criterion (AIC), Bayesian Information Criterion (BIC), and the Minimum Description Length (MDL) score (see Heckerman and coworkers ${ }^{51}$; Friedman and Goldszmidt $^{52}$ ). The search for the best scoring network is computationally very expensive as the number of possible DAGs is superexponential in the number of nodes (genes) considered and therefore ad hoc random searches are usually used.

Recent work by two of the authors (Almudevar and Salzman, submitted for publication) suggests that a score suitable for learning a single network (that is, a maximum scoring network) may be unsuitable for use in Bayesian averaging. Both the BIC and AIC score are of the form

$$
L(G) \exp (\lambda c(G))
$$

where $c(G)$ is the number of free parameters in $f$ (i.e., parametric complexity), which is determined by $G$, and $\lambda$ may be interpreted as a complexity penalty, set to -1 for AIC and $-0.5 \log (n)$ for $n$ replications, for BIC. We may alternatively vary $\lambda$ to obtain a family of scores. Computer experiments suggest that, where a good estimate of graph complexity can be obtained by maximizing BIC, when used as a posterior density, the complexity estimate produced by Bayesian averaging can be considerably higher. It is possible, however, to select $\lambda$, which results in a Bayesian estimate of complexity that matches the more accurate maximum BIC estimate. The resulting Bayesian averaging method better controls for spurious complexity, with little effect on the inference of true features.

\section{CONCLUSION}

In this article, we emphasize that measures of differential expression across experimental conditions are fundamentally different from measures of coexpression, usually captured as correlation coefficients. They each provide different forms of information, and, in fact, a full understanding of differential expression is unlikely in the absence of a consideration of both types of information.

We also give a survey of the theory of correlation as applied to the reconstruction of graphical representations of gene regulatory networks, as well as some of the statistical methodologies used to implement these ideas. Although theoretical limits exist regarding which control laws can be recovered in the absence of specialized experiments, coexpression measures can and are being used to uncover significant features of cellular control.

Acknowledgments: The research of A. Almudevar, P. Salzman, and A. Yakovlev is supported by National Institutes of Health/National Institute of General Medical Sciences Grant
GM075299. We thank Dr. Timothy Mhyre for many fruitful discussions.

\section{REFERENCES}

1. Storey JD. Comment on Resampling-based multiple testing for DNA microarray data analysis by Ge, Dudoit, and Speed. Test 12:1-77, 2003.

2. Qiu X, Brooks A, Klebanov L, Yakovlev A. The effects of normalization on the correlation structure of microarray data. BMC Bioinformatics 6:120, 2005.

3. Friedman N, Linial M, Nachman I, Pe'er D. Using Bayesian networks to analyze expression data. J Comput Biol 7:601-620, 2000.

4. Wolfinger RD, Gibson G, Wolfinger ED, Bennett L, Hamadeh H, Bushel P, et al. Assessing gene significance from cDNA microarray expression data via mixed models. J Comp Biol 8:625-637, 2001.

5. Dudoit S, Shaffer JP, Boldrick JC. Multiple hypothesis testing in microarray experiments. Stat Sci 18:71-103, 2003.

6. Simon RM, Korn EL, McShane LM, Radmacher MD, Wright GW, Zhao Y. Design and analysis of DNA microarray investigations. New York: Springer, 2003.

7. Speed TP. Statistical analysis of gene expression microarray data. Boca Raton, FL: Chapman \& Hall CRC, 2003.

8. Lee M-L. Analysis of microarray gene expression data. Boston: Kluwer, 2004.

9. McLachlan GL, Do K-A, Ambroise C. Analyzing microarray gene expression data. Hoboken, NJ: Wiley, 2004.

10. Wit E, MacClure. J Statistics for microarrays. Chichester: Wiley, 2004.

11. Tian L, Greenberg SA, Kong SW, Altschuler J, Kohane IS, Park PJ. Discovering statistically significant pathways in expression profiling studies. Proc Natl Acad Sci USA 102:13544-13549, 2005.

12. Ma'ayan A, Gardiner K, Iyengar R. The cognitive phenotype of Down syndrome: insights from intracellular network analysis. NeuroRx 3:394-403, 2006.

13. Ginsberg SD, Che S, Counts SE, Mufson EJ. Single cell gene expression profiling in Alzheimer's disease. NeuroRx 3:302-317, 2006.

14. Miller RM, Federoff HJ. Microarrays in Parkinson's disease: a systematic approach. NeuroRx 3:318-325, 2006.

15. Klebanov L, Jordan C, Yakovlev A. A new type of stochastic dependence revealed in gene expression data. Stat Appl Genet Mol Biol 5:7, 2006

16. Butte AJ, Tamayo P, Slonim D, Golub TR. Discovering functional relationships between RNA expression and chemotherapeutic susceptibility using relevance networks. Proc Natl Acad Sci USA 97:12182-12186, 2000.

17. Qiu X, Klebanov L, Yakovlev AY. Correlation between gene expression levels and limitations of the empirical Bayes methodology for finding differentially expressed genes. Stat Appl Genet Mol Biol 4:34, 2005.

18. Qiu X, Yakovlev A. Instability of false discovery rate estimation. Technical Report 06/03. Available at: http://www.urmc.rochester.edu/ smd/biostat/people/faculty/andrei.htm. Accessed: 2006.

19. Goerman JJ, van de Geer SA, de Kort F, van Houwelingen HC. A global test for groups of genes: testing association with a clinical outcome. Bioinformatics 20:93-99, 2004.

20. Jaeger J, Sengupta R, Ruzzo WL. Improved gene selection for classification of microarrays. Kauai, HI: Pacific Symposium on Biocomputing, 2003

21. Xiao Y, Frisina R, Gordon A, Klebanov L, Yakovlev A. Multivariate search for differentially expressed gene combinations. BMC Bioinformatics 5:164, 2004.

22. Lu Y, Liu P-Y, Deng H-W. Hotelling's $T^{2}$ multivariate profiling for detecting differential expression in microarrays. Bioinformatics 21:3105-3113, 2005.

23. Dettling M, Gabrielson E, Parmigiani G. Searching for differentially expressed gene combinations. Genome Biol 6:R88, 2005.

24. Efron B. Correlation and large-scale simultaneous significance testing. Available at: http://www-stat.stanford.edu/ brad/papers/. Accessed: 2006. 
25. Eisen MB, Spellman PT, Brown PO, Botstein D. Cluster analysis and display of genome-wide expression patterns. Proc Natl Acad Sci USA 95:14863-14868, 1998.

26. Lee M-L, Kuo FC, Whitmore GA, Sklar J. Importance of replication in microarray gene expression studies: statistical methods and evidence from repetitive DNA hybridizations. Proc Natl Acad Sci USA 97:9834-9839, 2000.

27. Pavlidis P, Li Q, Noble WS. The effect of replication on gene expression microarray experiments. Bioinformatics 19:1620$1627,2003$.

28. Qiu X, Xiao Y, Gordon A, Yakovlev A. Assessing stability of gene selection in microarray data analysis. BMC Bioinformatics 7:50, 2006.

29. Lee M-LT, Gray RJ, Björkbacka H, Freeman MW. Generalized rank tests for replicated microarray data. Stat Appl Genet Mol Biol 4:3, 2005

30. Klebanov L, Gordon A, Xiao Y, Land H, Yakovlev A. A permutation test motivated by microarray data analysis. Comp Stat Data Anal (in press).

31. Xiao Y, Gordon A, Yakovlev A. The $\mathrm{L}_{1}$-version of the Cramer-von Mises test for two-sample comparisons in microarray data analysis. Technical Report 06/03. Available at: http://www.urmc.rochester.edu/ smd/biostat/people/faculty/andrei.htm. Accessed: 2006.

32. Baldi P, Hatfield GW. DNA microarrays and gene expression: from experiments to data analysis and modeling. Cambridge, UK: Cambridge University Press, 2002.

33. Verma TS, Pearl J. Equivalence and synthesis of causal models. Proc UAI 6:255-268, 1990.

34. de la Fuente A, Bing N, Hoeschele I, Mendes P. Discovery of meaningful associations in genomic data using partial correlation coefficients. Bioinformatics 20:3565-3574, 2004.

35. Schafer J, Strimmer K. An empirical Bayes approach to inferring large-scale gene association networks. Bioinformatics 21:754764, 2005.

36. Wille A, Bühlmann P. Low-order conditional independence graphs for inferring genetic networks. Stat Appl Genet Mol Biol 5(1) Article 1. 2006. Available at: http://www.bepress.com/sagmb/ vol5/iss1/art1. Accessed: 2006.

37. Butte AJ, Kohane IS. Mutual information relevance networks: functional genomic clustering using pairwise entropy measurements. Pacific Symp Biocomput 418-429, 2000.
38. Chu T, Glymour C, Scheines R, Spirtes P. A statistical problem for inference to regulatory structure from associations of gene expression measurements with microarrays. Bioinformatics 19:11471152, 2003.

39. Bahcall OG. Single cell resolution in regulation of gene expression. Mol Syst Biol 1:41000, 2005.

40. Zhou X, Kao M-CJ, Wong WH. Transitive functional annotation by shortest-path analysis of gene expression data. Proc Natl Acad Sci USA 99:12783-12788, 2002.

41. Jordan MI (ed). Learning in graphical models. Cambridge, MA: The MIT Press, 1998.

42. Madigan D, York J. Bayesian graphical models for discrete data. Int Stat Rev 63:215-232, 1995.

43. Hoeting JA, Madigan D, Raftery AE, Volinsky CT. Bayesian model averaging: a tutorial. Stat Sci 14:382-417, 1999.

44. Friedman N, Koller D. Being Bayesian about Bayesian network structure: a Bayesian approach to structure discovery in Bayesian networks. Machine Learn 50:95-125, 2003.

45. Dash D, Cooper GF. Model averaging for prediction with discrete Bayesian networks. J Machine Learn Res 5:1177-1203, 2004

46. Almudevar, A, Salzman P. Using a Bayesian posterior density in the design of perturbation experiments. In Proceedings of the IEEE Symposium on Computational Intelligence and Bioinformatics and Computational Biology, 2005, San Diego, CA, 2005.

47. Pearl J. Probabilistic reasoning in intelligent systems. San Francisco, CA: Morgan Kaufmann, 1988.

48. Friedman N. Inferring cellular networks using probabilistic graphical models. Science 303:799-805.

49. Sebastiani P, Abad M, Ramoni MF. Bayesian networks for genomic analysis. In: Genomic signal processing and statistics, EURASIP Book series on signal processing and communications (Dougherty ER, Shmulevich I, Chen J, Wang ZJ, eds), Ch 8, pp 281-320. New York: Hindawi Publishing Corporation, 2005.

50. Hartemink AJ. Reverse engineering gene regulatory networks. Nat Biotechnol 23:554-555, 2005.

51. Heckerman D, Geiger D, Chickering DM. Learning Bayesian networks: the combination of knowledge and statistical data. Machine Learn 20:197-243, 1995.

52. Friedman N, Goldszmidt M. Learning Bayesian networks with local structure. In: Learning in graphical models (Jordon MI, ed), pp 412-459. Cambridge, MA: The MIT Press, 1998. 